\title{
TRANSVERSE ENERGY AND MULTIPLICITY DISTRIBUTIONS OF p-p AND A+A INTERACTIONS
}

Michael J. TANNENBAUM and the E802 Collaboration

BNL --44885

Brookhaven National Laboratory, Upton NY 11973, USA

DE90 015686

Data from AGS-E802 have been presented at the recent HIPAGS warkshop at BNL ${ }^{1-}$, published in journals ${ }^{2,3,4}$ and in conference proceedings (St. $\mathrm{Malo}^{5}$, Moriond ${ }^{6}$ ), to which the reade: is referred. Two results are mentioned here. The Lead Glass distribution $d E_{T}^{0} / d \eta$ from central $\mathrm{O}+\mathrm{Au}$ collisions ${ }^{5}$ can be coinpared to the spectrometer $d n\left(\pi^{-}\right) / d y$ from central Si+Au collisions ${ }^{4}$. The agreement of the two distributions is excellent and confirms that the estimated ${ }^{5,6}$ position and value of the maximum in the distribution $\left.\eta\right|_{\max }, d E_{T} /\left.d \eta\right|_{\max }$, and the width $\Delta \eta_{F W H M}$, from the $\mathrm{PbGl}$ data are reasonable, and that nothing exceptional is occurring outside of the $\mathrm{PbGl}$ acceptance. Additionally, these data, and previous measurements of pseudorapidity distributions of multiplicity and Transverse Energy at both the AGS and CERN are analyzed in an acceptance-independent and model-independent method, with the conclusion that simple considerations of nuclear geometry do not provide an explanation of the different $\sqrt{s_{N N}}$ depenciences observed in ${ }^{16} \mathrm{O}+\mathrm{Au}$ and p-p reactions.

\section{1. "TRANSVERSE ENERGY" AND MULTIPLICITY DISTRIBUTIONS}

Transverse Energy $\left(E_{T}\right)$ distributions have recently become a respected analytical tool for soft multiparticle collisions, particularly in nucleus-nucleus reactions. It has been shown, by experiments ${ }^{2,3,7,8,9,10}$ at $\mathrm{BNL}$ and $\mathrm{CERN}$, that $E_{T}$ distributions from nuclear collisions are largely dominated by the nuclear geometry, at the present level of sensitivity. However, it is possible that dynamical effects also play a role.

Pseudorapidity distributions of transverse energy, $d E_{T} / d \eta$, are important in the discussion of "nuclear stopping" because one of the simplest concepts of stopping is that the projectile and target "participants" merge into a hadronic "fireball", which then decays isotropically ${ }^{7,8,9,10}$. This would be quite distinct from the normal behavior of the "soft" multiparticle production processes that predominate in high energy collisions, in which produced particles have limted transverse momentum $\left(\left\langle p_{T}\right\rangle \simeq \frac{1}{3} \mathrm{GeV} / \mathrm{c}\right)$ and very broad and flat rapidity distributions, a situation usually described as "longitudinal phase space". In this limit, an $E_{T}$ measurement is simply an analog method of counting particles: each particle produced has roughly the same value of $E_{i} \sin \theta_{i} \simeq\left\langle p_{T}\right\rangle$.

This manuscript has been authored under contract number DE-AC02-76CH00016 with the U.S. Department of Energy.

Poster Session, to appear in the QM'90 proceedings 


\section{E802 LEAD GLASS MEASUREMENTS}

As part of AGS Experiment 802, an array of 245 Lead Glass blocks, covering half the azimuth, with a polar angular acceptance approximately $8^{\circ} \leq \theta \leq 32^{\circ}$, which corresponds roughly to a laboratory pseudo rapidity range $1.25 \leq \eta \leq 2.50$ with good acceptance, when edges and corners are taken into account, measured the transverse energy $\left(E_{T}\right)$ distributions from primary beams of protons, ${ }^{16} \mathrm{O}$, and ${ }^{28} \mathrm{Si}$, at $14.6 \mathrm{~A} \mathrm{Gev} / \mathrm{c}$, incident on targets of $\mathrm{Be}, \mathrm{Al}$, $\mathrm{Cu}$ and $\mathrm{Au}$. The lead glass is most sensitive to electromagnetic radiation, but also responds to relativistic charged hadrons $(\beta>0.8)$. No attempt is made to correct for the Cerenkov light from charged hadrons on an event by event basis because an incalculably large pointby-point systematic error would have to be assigned. The response of the $\mathrm{PbGl}$ to Cerenkov light is linear to $<1 \%$ whatever the source, so that the total detector response provides a good measure of the global pion yield in the central rapidity region of these reactions ${ }^{2}$.

Data from E802 have been presented at the recent HIPAGS workshop at BNL ${ }^{1}$, published in journals ${ }^{2,3,4}$ and in conference proceedings (St. Malo ${ }^{5}$, Moriond ${ }^{6}$ ), to which the reader is referred. Also, correlations among the 4 detector systems in E802 have been shown to be a powerful diagnostic $\mathrm{ool}^{1,5}$. In this brief discussion, some of these results are highlighted and compared. In figure 1 , the pseudorapidity distribution in the Lead Glass, $d E_{T}^{0} / d \eta$, from central $\mathrm{O}+\mathrm{Au}$ collisions ${ }^{5}$ (diamonds) is compared to the spectrometer $d n\left(\pi^{-}\right) / d y$ from central $\mathrm{Si}+\mathrm{Au}$ collisions ${ }^{4}$ (squares). The agreement of the two distributions is excellent and confirms that the estimated ${ }^{5,6}$ position and value of the maximum in the distribution $\left.\eta\right|_{\max }, d E_{T} /\left.d \eta\right|_{\max }$, and the full width at half maximum $\Delta \eta_{F W H M}$, from the PbGl data are reasonable, and that nothing exceptional is occurring outside of the $\mathrm{PbGl}$ acceptance. It is evident that the parameters $\left.\eta\right|_{\text {max }}, d E_{T} /\left.d \eta\right|_{\max }$, and $\Delta \eta_{F W H M}$, provide a simple and complete characterization of the measured (pseudo)rapidity distributions $d E_{T} / d \eta$ and $d n / d y$ in a relatively acceptance-independent and model-independent fashion.

\section{ACCEPTANCE-INDEPENDENT COMPARISON OF ALL EXPERIMENTS}

The simple characterization of pseudorapidity distributions, illustrated above, allows a systematic comparison of $E_{T}$ measurements from all the RHI experiments, in spite of the fact that the detectors cover nearly disjoint regions of pseudorapidity: WA809, E814 ${ }^{10}$ forward; NA34(Helios) ${ }^{8}$-backward; $\mathrm{E} 802^{2,4}, \mathrm{NA35}{ }^{7}$-central. It is possible to compare these experiments independently of acceptance by first estimating $E_{T}^{\text {cent }}$, the $E_{T}$ observed in "central collisions", using the NA34-Helios" definition (a factor of two in cross section down from the "knee" of the distribution), for all experiments. Then, the value of $d E_{T}^{c e n t} /\left.d \eta\right|_{\max }$ at the maximum in the pseudorapidity distribution can be estimated ${ }^{6,11}$. The results are shown in fig. 2, as a function of the nucleon-nucleon c.m.energy $\sqrt{s_{N N}}$ for ${ }^{16} O+A u(W)$ reactions. It is appropriate to compare the $\sqrt{s_{N N}}$ dependence of $d E_{T}^{c e n t} /\left.d \eta\right|_{\max }$ to measurements of $d n /\left.d y\right|_{\text {max }}$ from $p-p$ collisions. The broken line on the figure is $16 \times d n /\left.d \eta\right|_{y=m} ^{N N}$ for $p-p$ collisions $^{32}$. If geometry were the only nuclaar effect, then the $\sqrt{s_{N N}}$ dependences would be identical in the $p-p$ and the ${ }^{16} \mathrm{O}+\mathrm{Au}$ reactions. However, $d E_{T}^{\text {cent }} /\left.d \eta\right|_{\max }$ in ${ }^{16} O+A u$ 


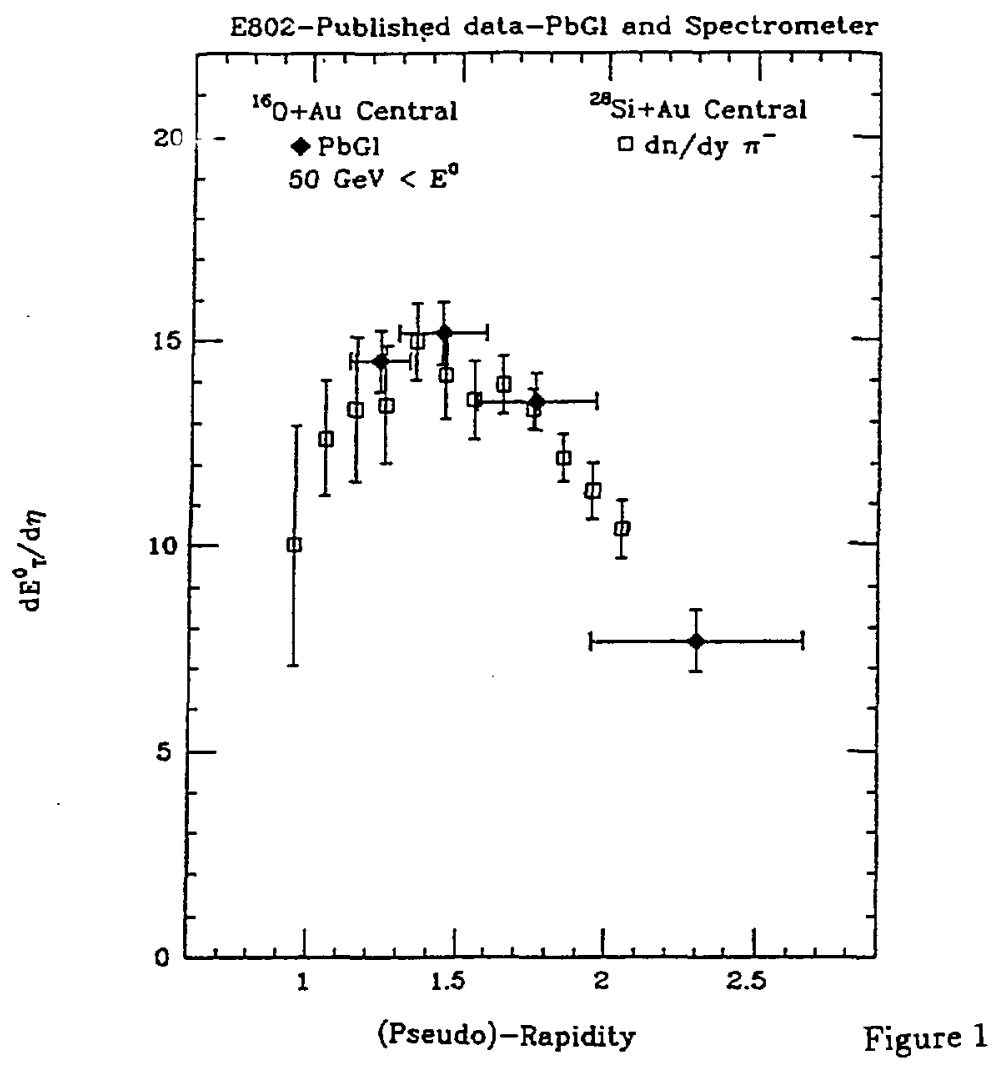

Maximum $d E_{\mathrm{T}} / \mathrm{d} \eta$ for "central" collisions

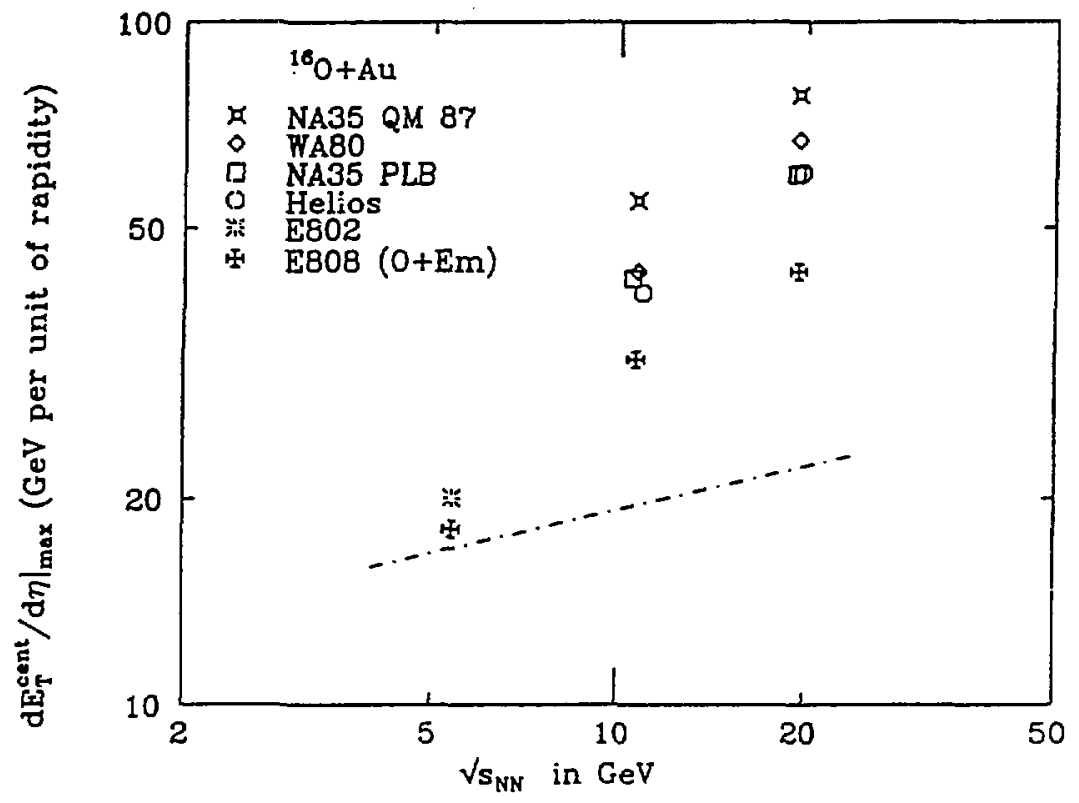

Figure 2 
increases by a factor of 3 from $\sqrt{s_{N N}}=5.4$ to $19.4 \mathrm{GeV}$, which is much greater than the corresponding increase of a factor of 1.31 of the multiplicity density in $p-p$ collisions-a relative increase of $3 / 1.3=2.3$.

The estimates of $E_{T}^{\text {cent }}$ are clearly dominated by systematic effects, the moșt notable being between the two NA35 measurements ${ }^{\top}$. However, a similar conclusion on the relative $\sqrt{s_{N N}}$ dependence of nucleus-nucleus and $p-p$ collisions can be drawn from the emulsion data of $\mathrm{E} 808^{13}$, where a direct comparison of pseudorapidity distributions for ${ }^{16} \mathrm{O}+\mathrm{AgBr}$ central collisions at $14.5 \mathrm{GeV} / \mathrm{c}$ per nucleon, $60 \mathrm{GeV} /$ nucleon and $200 \mathrm{GeV} /$ nucleon is available for charged particle multiplicity. The maximum in the multiplicity distribution for "shower particles" $(\beta>0.7), d n_{s} /\left.d \eta\right|_{\max }$, increases by a factor of 2.4 from $\sqrt{s_{N N}}=5.4$ to $19.4 \mathrm{GeV}$, an increase of a factor of 1.8 relative to $p-p$.

It is evident from these considerations, that systematic comparision of the data from nucleus-nucleus, $p+A$, and $p-p$ interactions at AGS and CERN energies can lead to quantitative conclusions, without detailed recourse to particular models. Simple considerations of nuclear geometry do not provide an explanation of the different $\sqrt{s_{N N}}$ dependences observed in ${ }^{16} \mathrm{O}+A u$ and p-p reactions. Dynamical effects, such as "energy stopping" at AGS energies and "transparency" at CERN energies, are also required.

\section{REFERENCES}

1. HIPAGS Workshop at BNL, March 5-7, 1990, to appear as BNL report.

2. E802 Collaboration, T. Abbott et al., Phys. Lett. B197 (1987) 285.

3. E802 Collaboration, Z. Phys. C38 (1988) 35,135.

4. E802 Collaboration, T. Abbott et al., Phys. Rev. Letters 64 (1990) 847.

5. M.J. Tannenbaum, Nucl. Phys. A488 (1988) 555c.

6. M.J. Tannenbaum et al., Proceedings of the 24th Rencontre de Moriond, ed. J. Tran Thanh Van, (Editions Frontieres, Gif-sur-Yvette, France, 1989), pp 581-586.

7. NA35 Collaboration, A.Bamberger, et al., Phys. Lett. B184 (1987) 271; W.Heck, et al., Z. Phys C38 (1988) 19.

8. NA34(Helios) Collaboration, Z. Phys. C38 (1988) 383; Phys. Lett. B214 (1988) 295.

9. WA80 Collaboration, S.P.Sorensen et al., Z. Phys. C38 (1988) 3,51; R.Albrecht, et al., Phys. Lett. B199 (1987) 297.

10. E814 Collaboration, P. Braun-Munzinger et al., Z. Phys. C38 (1988) 45.

11. M.J.Tannenbaum, International Journal of Modern Physics A 4(1989) 3377.

12. The parametrization for $\mathrm{p}-\mathrm{p}$ is $d n /\left.d \eta\right|_{\max }=0.74(\sqrt{s})^{0.210}$, where the $\sqrt{s}$ is in $\mathrm{GeV}$. UA5 Collaboration, Z. Phys. C33 (1986) 1.

13. E808 Collaboration, H. von Gersdorff, et al., Phys. Rev. C39 (1989) 1385.

\section{DISCLAIMER}

This report was prepared as an account of work sponsored by an agency of the United States Government. Neither the United States Government nor any agency thereof, nor any of their employees, makes any warranty, express or implied, or assumes any legal liability or responsibility for the accuracy, completeness, or usefulness of any information, apparatus, product, or process disclosed, or represents that its use would not infringe privately owned rights. Reference herein to any specific commercial product, process, or service by trade name, trademark, manufacturer, or otherwise does not necessarily constitute or imply its endorsement, recommendation, or favoring by the United States Government or any agency thereof. The views and opinions of authors expressed berein do not necessarily state or reflect those of the United States Government or any agency thereof. 